\title{
Fútbol y redes sociales. Análisis de la gestión de Relaciones Públicas 2.0 por los clubes de fútbol
}

\author{
Antonio Castillo EsParcia \\ Universidad de Málaga \\ acastilloe@uma.es
María Jesús Fernández TORRES
Universidad de Málaga
mariajesusfernandez@uma.es \\ Elizabeth CASTILLERo Ostío \\ Universidad de Málaga \\ elizabethcastillero@hotmail.com
}

Recibido: 20 de marzo de 2015

Aceptado: 28 de septiembre de 2015

\section{Resumen}

Las relaciones públicas se configuran como una actividad esencial en la gestión de la comunicación con los públicos de los clubes de fútbol ya que son muy activos y su grado de reactividad es alto. Con este texto se analiza el papel que desempeñan las redes sociales en los clubes de fútbol que poseen mayor número de ingresos. Para ello, se desarrolla una metodología que estudia qué presencia tienen en las redes sociales, el número de seguidores, qué grado de interacción se produce entre clubes y públicos y los contenidos y las temáticas de los textos de las redes sociales. Los resultados muestran un público activo pero con intervenciones relacionadas con los resultados futbolísticos y una gestión comunicativa unidireccional por parte de los clubes.

Palabras clave: relaciones públicas, redes sociales, fútbol, comunicación digital.

\section{Football and Social Network. Analysis of the management of Public Relations 2.0 by Football Clubs}

\begin{abstract}
The public relations are formed as a assential activity in the management of the communication with the audience of the football clubes, due to they are very active and its degree of resactivity is big too. In this text it is analized the function that the social network executes in the football clubes which own the greatest number of deposit. In order to do that, it is developed a methodology that studies what is the presence they have in the social network, the number of fans, what is the interaction degree that is produced between clubes and the audience and the contents and topics of the text in social network. The results show an active audience, but with interventions related to football results and a communicative, one way management from the clubes.
\end{abstract}

Keywords: public relations, social network, football, digital communication.

\section{Referencia normalizada}

CASTILLO ESPARCIA, Antonio; FERNÁNDEZ TORRES, María Jesús; y CASTILLERO OSTÍO, Elizabeth: "Fútbol y redes sociales. Análisis de la gestión de Relaciones Públicas 2.0 por los clubes de fútbol”. Estudios sobre el Mensaje Periodístico. Vol. 22, Núm. 1 (enero-junio), págs.: 239-254. Madrid, Ediciones Complutense.

Sumario: 1. Introducción; 1.1. Planificación estratégica de las Relaciones Públicas 2.0. 2. Metodología. 3. Resultados; 3.1. Temáticas de las diferentes redes sociales. 4. Conclusiones. 5. Referencias bibliográficas. 


\section{Introducción}

La importancia y la repercusión social del fútbol conlleva la confluencia de intereses complejos y diversos. Implicaciones políticas, sociales, económicas y culturales son inherentes a la comunicación del fútbol y esas relaciones suponen un foco permanente de los medios de comunicación sobre las entidades futbolísticas y sus actores. En este sentido, Meneses y Avalos señalan que: El deporte y el futbol mediáticos que atraen la atención de periodistas, cámaras y una amplia gama de televidentes, lectores y radioyentes que aseguran significativas cuotas de rating y de mercado publicitario, constituyen una realidad deportiva promovida por complejas alianzas de intereses que vinculan principalmente a las federaciones, los clubes, los estados y las empresas o los Medios de Comunicación.

Consecuencia de ello, el fútbol, las entidades deportivas, los clubes mantienen una presencia constante y permanente en el ecosistema comunicativo. Eso supone que la gestión comunicativa debe desarrollarse como comunicación de organización por lo que el papel de las relaciones públicas en los clubes de fútbol es inmanente. Todo club de fútbol debe ser capaz de gestionar, desde la dirección, una estrategia de relaciones públicas que le permita planificar, desarrollar, ejecutar y evaluar la comunicación. Ya resulta añejo una perspectiva estrictamente mediática del fútbol en la que los medios de comunicación son los únicos intermediarios entre organización (club de fútbol) y público (aficionados). Las relaciones con los públicos (public relations) de los clubes de fútbol abarcan informaciones sobre la entidad a través de los medios de comunicación, pero también las empresas patrocinadoras comunican, los empleados (llamados futbolistas) comunican, los aficionados comunican y los medios comunicación. Nos encontramos en una metacomunicación de la comunicación con continuas autorreferencias ya sea cuando el club habla de sí mismo, cuando los futbolistas gestionan su imagen y cuando las empresas patrocinan a clubes, futbolistas e incluso estadios (Arceo, 2003 y Ginesta, 2010).

Cuando se habla de comunicación para los clubes nos encontramos con los siguientes actores:

- La propia empresa que gestiona su comunicación a través de sus estructuras de comunicación. Esos gabinetes de comunicación son los encargados de las relaciones mediáticas y su función se expande no solo a los medios de comunicación sino también la presencia en las redes sociales, a la página web de la entidad, a la asistencia a actos institucionales y deportivos. Es decir, todo un conjunto de acciones de comunicación que son las propias de una gran empresa de la actualidad.

- Los propios empleados y asalariados de la entidad. Realmente son empleados con una particularidad y notoriedad que va más allá de su relación laboral. Esos ídolos de masas sobrepasan y sobresalen de su empresa ya que gestionan su imagen personal. Su comportamiento, en muchas ocasiones, es el de actuar como verdaderas empresas que tienen sus propios objetivos y así pueden gestionar los denominados derechos de imagen y realizar acciones empresariales sobre otros sectores productivos (Minquet, 2004). 
- Las múltiples páginas web y espacios en las redes sociales de aficionados que como seguidores del club y de los futbolistas gestionan una comunicación que, en cierta medida afecta a la comunicación de los clubes. Se convierten en muchas ocasiones en amplificadores de informaciones.

- Los medios de comunicación como transmisores de informaciones sobre los clubes de fútbol, sobre la competición y sobre los actores que participan (futbolistas, presidentes, aficionados, entrenadores, organizaciones deportivas nacionales e internacionales). Nos referimos a los medios de comunicación de información general pero también a los especializados (Olabe, 2011). En este sentido, los medios de comunicación desempeñan un papel esencial ya que "hay, pues, una estrecha relación entre el número de acontecimientos deportivos que se disputan semanalmente y el número de unidades de información deportiva que aparecen en los medios" (González, 2004: 274).

- Los periodistas como actores del quehacer comunicativo ya sea desde una perspectiva informativa pero también opinativa (Castillo, 2002). La imbricación entre periodistas-seguidor de un club es una variante normalizada, asumida y regularizada. El grado de personalidad de periodistas y defensor de los colores de una camiseta es algo usual en los clubes de fútbol, algo que ocurre en muy pocos deportes y en otros ámbitos de la comunicación.

- Los aficionados a los clubes que van más allá de una función como meros consumidores de los productos de la empresa. Esa total imbricación entre club y seguidor nos muestra unos consumidores permanentes, fieles, compulsivos y fanáticos. Esto es, su relación no se rompe (puede tener altibajos e intermitencias), nace y se mantiene (no cambia a otro producto u otro club) y es esencialmente pasional para los bueno (euforia) y para lo malo (críticas). Es una relación extremista.

- Instituciones políticas como asociación entre comportamientos de identidad nacional y la instrumentalización política de elementos identitarios. Las asimilaciones entre clubes de fútbol y selecciones nacionales e identidades es uno de los aspectos más relevantes de la comunicación futbolística (González, 2014, Roldán, 2007, Ginesta y San Eugenio, 2013; Xifra, 2008). Tal como señala L'Etang la imbricación entre cultura y deporte es muy estrecha "Sport is also a sub-cultural or micro-cultural element of both national, ethnic and organizational cultures. National leaders use sport to enhance national identity and to endeavour to unify national culture" (L'Etang, 169).

Todos estos actores confluyen generando un ecosistema comunicativo de muchas implicaciones, con influencias recíprocas, de índole centrípeta y centrifuga a partir de un resultado (pero no siempre) y que dificulta la estrategia de comunicación. Por ello, los responsables de comunicación deben saber modular sobremanera las acciones de comunicación al socaire del ecosistema comunicativo relacionado con los clubes y sus implicaciones comunicativas. Así, una declaración inoportuna, un resultado negativo, un conflicto interno influye en los procesos de gestión de la comunicación. Y eso supone una imprevisibilidad de acontecimientos que se añaden a la sobreexposi- 
ción comunicativa de los clubes de fútbol y de sus públicos implicados. Todo ello supone la necesidad de que se realicen acciones permanentes de evaluación de la comunicación para poder interactuar con el entorno.

\subsection{Planificación estratégica de las Relaciones Públicas 2.0}

El estudio de las estrategias de comunicación digitales se ha desarrollado a partir de la mejora de los procesos interactivos en las organizaciones. La irrupción de la planificación estratégica en relaciones públicas en las organizaciones supone reconocer el papel de la comunicación en los procesos de dirección estratégica (Matilla, 2011). Y esa dirección estratégica implica el reconocimiento de la labor de las relaciones públicas como vectores de la comunicación en su doble actividad de relación y de engagement (Vercic, Verhoeven y Zerfass, 2014 y Verhoeven, Tench, Zerfass y Moreno (2012). Almansa y Castillo-Esparcia (2014:24) apuntan que la aplicación de las tecnologías ha supuesto un cambio en los modos de la comunicación y de las relaciones públicas en las organizaciones:

"Pero las nuevas tecnologías e internet no aportan únicamente mejoras en la gestión de la comunicación. Los sitios webs han supuesto grandes ventajas y oportunidades para quienes gestionan la comunicación".

Cuando se intenta delimitar la definición de relaciones públicas 2.0 es necesario apelar a los siguientes elementos característicos:

- Incertidumbre sobre la gestión de las relaciones puesto que solo podemos gestionar las acciones de comunicación que están promovidas desde la organización.

- Bilateralidad o simetría entre organización y públicos o públicos y organización debido a que estos dos actores poseen capacidad de creación y generación de acciones de comunicación, esto es, son al mismo tiempo, generadores y receptores de información.

- Los públicos son complejos y multifactoriales como consecuencia de que la red coadyuva a que se puedan generar acciones comunicativas prácticamente por parte de cualquier actor. Empero, esa potencialidad no se hace efectiva sino que solo los individuos, los grupos organizados o sensibilizados son los que toman en consideración acciones activas. Eso supone una pluralidad individual y asociativa en la que los más activos, organizados y sensibilizados son los que planifican y ejecutan acciones comunicativas.

- Viralidad como elemento de transmisión de las iniciativas y de los mensajes en un sistema de replicación de acciones y reacciones recíprocas entre los sujetos participantes (organizaciones con organizaciones, organizaciones con individuos, individuos con organizaciones e individuos con individuos).

- Implicación de todos los actores que participan en el ecosistema comunicativo digital ya que el escenario nos obliga a participar en las redes comunicativas cuando algunos de los actores nos hace partícipes. Ello exige una correcta pla- 
nificación de la comunicación, el permanente monitoreo de las redes (Castillo y Álvarez, 2014), la proactividad comunicativa y la creación de canales de comunicación bidireccionales. Lo contrario a estos aspectos supone una pérdida de la iniciativa, empeorar las relaciones con los públicos y exponerse a permanentes campañas de comunicación negativas.

- Multimedialidad como necesidad de que los mensajes lleguen en sonido, imagen y texto de manera separada y conjunta.

Sin embargo, los estudios sobre comunicación de los clubes de fútbol nos muestran una escasa actividad en la bidireccionalidad. Así, para Sotelo "las webs se utilizan como canal de comunicación unidireccional, ofrecen noticias del club y comercialización de productos y servicios, pero permiten aún poca interacción por parte de los aficionados. Y lo mismo sucede con su presencia en las redes sociales online, utilizadas por las marcas, instituciones y deportistas de manera básicamente unidireccional, como canales para la propagación de sus comunicados offline. Muy pocos utilizan las redes allí donde son más potentes: para conversar con los seguidores". (Sotelo, 2012: 228)

El papel de la comunicación 2.0 ha sido fruto de un proceso de asentamiento de estrategias comunicativas adaptadas a los diferentes públicos. Así, Castillo-Esparcia y Almansa (2005: 137) señalan que las relaciones con los medios de comunicación a través de las salas de prensa virtuales suponen una mejora de la presencia mediática en las organizaciones. Esa etapa inicial, en la que se centraban en las relaciones con los medios, dio paso a una creciente inserción de técnicas 2.0 integrada en la evolución del ecosistema digital. Así, la expansión de medios interactivos supone la adaptación de las estrategias de comunicación a esas nuevas modalidades de relaciones con los diferentes públicos de las organizaciones. Y la evolución de los públicos demanda una modificación de las estrategias de comunicación, tal como señalan Almansa, Fonseca y Castillo-Esparcia, 2013: 129).

La comunicación de los clubes de fútbol debe ser gestionado desde la propia organización. Tal como apunta Almanza, "el FC Barcelona es el encargado de establecer los temas de la agenda mediática. Desde sus medios de comunicación oficiales coordina la información, el discurso y el tono de esas informaciones" (Almanza, 2013:17).

Así, la comunicación 2.0 se caracteriza por la incertidumbre, simetría, complejidad, viralidad, implicación y multimedialidad. Todo ello en aras de establecer relaciones mutuamente satisfactorias y de interés común con los públicos de las organizaciones. Ese relacionamiento -engagement- es el paso indefectible de las organizaciones que pretenden participar, compartir y empatizar en la sociedad y en el ecosistema comunicativo. Tal como señalan algunos estudios el uso de las tecnologías de la comunicación para la movilización social se ha convertido en una de las principales acciones de comunicación de las organizaciones sociales (Haro y Sampedro, 2011; Castillo-Esparcia, García y Smolak, 2013). 


\section{Metodología}

El siguiente estudio tiene como fin el análisis de la presencia en las redes sociales de los 20 clubes de fútbol con mayor volumen de facturación del mundo. La selección de estos es según los datos proporcionados por el estudio Football Money League 2014, elaborado por Deloitte.

Tabla 1. Clubes con mayor número de ingresos. Fuente: Deloitte.

\begin{tabular}{|l|r|l|r||}
\hline \multicolumn{1}{|c|}{ Clubes de fútbol } & $\begin{array}{c}\text { Miles de } \\
\text { euros }\end{array}$ & \multicolumn{1}{|c|}{ Clubes de fútbol } & $\begin{array}{c}\text { Miles de } \\
\text { euros }\end{array}$ \\
\hline Real Madrid & 518.9 & Borussia Dortmund & 256.2 \\
\hline F.C. Barcelona & 482.6 & Liverpool & 240.6 \\
\hline Bayern Munich & 431.2 & Schalke 04 & 198.2 \\
\hline Manchester United & 423.8 & Tottenham Hotspur & 172.0 \\
\hline París Saint-Germain & 398.8 & Inter de Milán & 168.8 \\
\hline Manchester City & 316.2 & Galatasaray & 157.0 \\
\hline Chelsea & 303.4 & Hamburgo & 135.4 \\
\hline Arsenal & 284.3 & Fenerbahçe & 126.4 \\
\hline Juventus & 272.4 & AS Roma & 124.4 \\
\hline \hline AC Milán & 263.5 & Atlético de Madrid & 120.0 \\
\hline
\end{tabular}

Se han tenido en cuenta para este análisis aquellas redes sociales recogidas en cada página web oficial de cada equipo analizado. También se han analizado aquellas redes sociales más populares y utilizadas por los clubes de fútbol investigados, como son Facebook, Twitter, Youtube, Google+ e Instagram.

Se ha decidido acotar el tiempo de análisis a siete días, desde el 15 de marzo de 2014 hasta el 21 de marzo de 2014.

La metodología utilizada ha sido el análisis de contenido mediante una plantilla de análisis, la cual se componía de los siguientes ítems:

1. Red social estudiada.

2. Número de seguidores, me gusta o suscriptores, permitiéndonos este dato ver cuáles son los clubes que cuentan con un número mayor de seguidores en las redes sociales.

3. Seguimiento a otras personas o instituciones, en el que caso en el que proceda, con el objetivo de visualizar los intereses del club y las relaciones que mantiene con el público gracias a la relación mantenida a través del seguimiento a otros.

4. Fecha de inicio en la red social, para comprobar si ya cuenta con una experiencia y una presencia importante en esta o su incorporación ha sido más tardía, por lo que no se encuentra todavía en un período de madurez y desarrollo en cuanto a la actividad detectada en la red.

5. Frecuencia de actualización de la red. Este ítem nos permite constatar si la página en la red social está creada simplemente para contar con una simple presencia o verdaderamente se utiliza con frecuencia, conllevando esto a la creación de un contacto más estrecho con la afición.

6. Comentarios de los seguidores. Estos permiten ver la naturaleza de estos y el vínculo que los une al club. 
Sobre la temática de las entradas ha sido analizada con una plantilla distinta con el objetivo de profundizar más en el contenido de la información publicada por el club de fútbol. Debido a que esta es muy variada, ha sido necesario su sistematización en diferentes ítems que se presentan como los temas más recogidos en cada red social. Consiguiendo con esto que la presentación de los datos sea más fácil de interpretar.

\section{Resultados}

Todos los clubes analizados disponen de presencia en las principales redes sociales como son Facebook, Twitter, Instagram y Google +. Eso implica que es la mínima presencia en esas cuatro redes sociales. En el caso de Youtube también encontramos una presencia masiva ya que están presentes todos los clubes menos Manchester United, Paris Saint-Germain, Manchester City, Borussia Dortmund y Galatasaray.

El club que mayor presencia tiene en las diferentes redes sociales es el FC Barcelona con una presencia en 10 redes diferentes, le siguen el Arsenal y el AC Milán con presencia en 8 redes sociales. Todos los clubes analizados realizan actividades en las principales redes sociales como son Facebook, Twitter y Youtube. En este último caso no poseen canal propio el Manchester United, el Paris Saint-Germain y el Manchester City. Todo ello supone una propensión adecuada a tener presencia en las redes sociales.

Tabla 2. Presencia de los clubes de fútbol en la red social Facebook.

Fuente: elaboración propia

\begin{tabular}{|c|c|c|c|c|}
\hline Facebook & $\begin{array}{l}\mathrm{N}^{0} \text { de } \mathrm{Me} \\
\text { gusta }\end{array}$ & $\begin{array}{l}\text { Fecha de inicio } \\
\text { en la red social }\end{array}$ & $\begin{array}{c}\text { Frecuencia de } \\
\text { actualización de la red }\end{array}$ & $\begin{array}{c}\text { Comentarios de } \\
\text { seguidores }\end{array}$ \\
\hline F.C. Barcelona & 58.414 .133 & $21 / 10 / 2009$ & A diario & $\begin{array}{c}\text { Apoyo al club y a los } \\
\text { jugadores } \\
\text { Opinión, críticas y } \\
\text { sugerencias }\end{array}$ \\
\hline Real Madrid & 54.643 .899 & $12 / 11 / 2007$ & A diario & Ídem \\
\hline Manchester United & 43.983 .910 & $\begin{array}{l}\text { No se tiene } \\
\text { constancia }\end{array}$ & A diario & Ídem \\
\hline Chelsea & 25.563 .197 & $\begin{array}{l}\text { No se tiene } \\
\text { constancia }\end{array}$ & A diario & Ídem \\
\hline Arsenal & 22.236 .001 & $\begin{array}{l}\text { No se tiene } \\
\text { constancia }\end{array}$ & A diario & Ídem \\
\hline AC Milán & 20.604 .923 & $02 / 11 / 2009$ & Cada 2 días & Ídem \\
\hline Liverpool & 16.933 .939 & $22 / 01 / 2013$ & A diario & Ídem \\
\hline Bayern Munich & 13.611 .277 & $12 / 07 / 2010$ & A diario & Ídem \\
\hline Juventus & 11.013 .894 & $27 / 04 / 2011$ & A diario & Ídem \\
\hline Manchester City & 10.491 .793 & $07 / 07 / 2009$ & A diario & Ídem \\
\hline Galatasaray & 10.334 .269 & $25 / 01 / 2011$ & A diario & Ídem \\
\hline París Saint-Germain & 8.718 .861 & $10 / 02 / 2013$ & Cada 2 días & Ídem \\
\hline Fenerbahçe & 7.809 .903 & $22 / 12 / 2010$ & A diario & Ídem \\
\hline Borussia Dortmund & 7.691 .221 & $08 / 06 / 2010$ & A diario & Ídem \\
\hline Tottenham Hotspur & 4.490 .318 & $03 / 03 / 2010$ & A diario & Ídem \\
\hline Inter de Milán & 3.558 .785 & $06 / 11 / 2010$ & A diario & Ídem \\
\hline AS Roma & 3.034 .320 & $10 / 11 / 2011$ & A diario & Ídem \\
\hline Atlético de Madrid & 2.619 .692 & $15 / 02 / 2010$ & A diario & Ídem \\
\hline Schalke 04 & 2.003 .057 & $\begin{array}{l}\text { No se tiene } \\
\text { constancia }\end{array}$ & A diario & Ídem \\
\hline Hamburgo & 622.600 & $24 / 04 / 2009$ & A diario & Ídem \\
\hline
\end{tabular}

Encabeza esta lista, por tener el mayor número de seguidores en esta red social, el equipo de fútbol del F.C. Barcelona, seguido del Real Madrid y el Manchester Uni- 
ted. Muy de lejos le sigue el Chelsea con menos de la mitad de aficionados que han marcado "me gusta" de los primeros equipos de esta lista.

Es necesario comentar que el ítem que recoge la información relativa a la fecha de iniciación de la actividad en esta red social no ha podido ser recogido en todos los casos. Esto se debe a que Facebook ofrece la posibilidad de ocultar esta información en la biografía, por lo que no siempre está visible. El club del Real Madrid es el que primero se inicia en esta red social, en 2007, con una diferencia de dos años de los siguientes equipos que lo hacen en 2009. Pero este hecho no ha sido determinante para posicionarlo en la primera posición en cuanto a número de seguidores, ya que este lo ocupa el F.C. Barcelona.

La mayoría de los clubs actualizan la información aparecida en la red a diario, y normalmente son varias las entradas cada día, a excepción de dos equipos que publican entradas cada 2 días: el AC Milán y el París Saint-Germain. Estos datos nos indican que los clubes utilizan con frecuencia esta red social para mantener informados a sus seguidores y establecer un vínculo bastante estrecho con ellos.

En cuanto a los comentarios que hacen los seguidores en estas páginas, una vez revisados todos, pueden agruparse de forma genérica en apoyo al club y a los jugadores, opiniones varias, críticas y sugerencias. Todo ello muy relacionado con actividades cotidianas y asociadas a los resultados deportivos. Así, nos encontramos que cuando se producen resultados positivos los comentarios son de alabanzas a los jugadores (esencialmente), mientras que los resultados negativos suponen críticas (por este orden, a entrenador, jugadores y directivos).

\subsection{Temáticas de las diferentes redes sociales}

Uno de los aspectos analizados eran las temáticas de las entradas en Facebook para conocer qué tipo de acontecimientos eran los más difundidos por los clubes que se han analizado. Con ello vamos a ver si imperan las acciones de tipo unidireccional o las bidireccionales.

Entre las actividades de tipo unidireccional se han considerado las siguientes: información sobre próximos partidos, textos sobre los partidos jugados, información sobre entrenamientos, opinión y entrevistas a jugadores y entrenador, productos del club, información sobre eventos publicitarios o de patrocinio, información de otros equipos del club, entre otros.

Las actividades que se han considerado como bidireccionales son petición de participación de los seguidores, sorteos/concursos, felicitaciones personales de cumpleaños de los seguidores, fotos e información de la afición.

Los resultados reflejan una mayoría de actividades unidireccionales generadas desde la propia entidad deportiva. Así, las acciones como informaciones sobre los partidos, la opinión de jugadores y las ruedas de prensa son los principales exponentes. Por el contrario, actividades bidireccionales como peticiones de participación de los seguidores, los sorteos/concursos o las fotografías remitidas por la afición cuentan con escasa incidencia.

La temática de las entradas en Facebook es muy variada, repitiéndose en la mayoría de los casos las publicaciones referentes a los partidos jugados, a los que están por 
jugar, a los entrenamientos, ruedas de prensa, entrevistas a jugadores y entrenadores, así como una petición por parte de los clubes de participación de los seguidores del equipo en la red social con opiniones en cuanto a preguntas y cuestiones propuestas en la red social.

Cabe comentar que en el caso de los dos equipos que lideran la lista, el F.C. Barcelona y el Real Madrid, son numerosas las fotografías sobre los entrenamientos de los jugadores subidas en esta red social, así como las referencias a los partidos clásicos protagonizados por estos dos equipos.

Entre los "otros temas" referenciados en esta red social encontramos: el referéndum por el nuevo espacio del F.C. Barcelona, información de los equipos en los medios de comunicación, recopilación de los mejores goles de los equipos, pronunciación de los clubes en cuanto a la celebración de días internacionales sobre alguna temática, recuerdo de partidos anteriores que se han convertido en "míticos" para el club, fotos de jugadores de los equipos, entrevistas a aficionados famosos del equipo y a antiguos jugadores, venta de entradas para los socios del club y estadísticas del equipo.

Tabla 3. Presencia de los clubes de fútbol en la red social Twitter. Fuente: elaboración propia

\begin{tabular}{|c|r|r|c|c|c|}
\hline Twitter & $\begin{array}{c}\text { No de } \\
\text { Seguidores }\end{array}$ & $\begin{array}{c}\text { Seguimiento } \\
\text { a otros }\end{array}$ & $\begin{array}{c}\text { Fecha de } \\
\text { inicio en la } \\
\text { red social }\end{array}$ & $\begin{array}{c}\text { Frecuencia de } \\
\text { actualización } \\
\text { de la red }\end{array}$ & $\begin{array}{c}\text { Comentarios de } \\
\text { seguidores } \\
\text { Real Madrid }\end{array}$ \\
10.437 .088 & 30 & $\begin{array}{c}\text { No se tiene } \\
\text { constancia }\end{array}$ & A diario & $\begin{array}{c}\text { Apoyo al club y a } \\
\text { los jugadores } \\
\text { Opinión, críticas } \\
\text { y sugerencias }\end{array}$ \\
\hline F.C. Barcelona & 6.173 .001 & 47 & Ídem & A diario & Ídem \\
\hline Galatasaray & 3.664 .817 & 4 & Ídem & A diario & Ídem \\
\hline Arsenal & 3.616 .872 & 163.488 & Ídem & A diario & Ídem \\
\hline Chelsea & 3.521 .330 & 291 & Ídem & A diario & Ídem \\
\hline Fenerbahçe & 2.933 .220 & 52 & Ídem & A diario & Ídem \\
\hline Liverpool & 2.459 .155 & 402.756 & Ídem & A diario & Ídem \\
\hline AC Milán & 1.828 .050 & 38 & Ídem & A diario & Ídem \\
\hline Manchester City & 1.584 .656 & 57.531 & Ídem & A diario & Ídem \\
\hline Juventus & 1.048 .344 & 40 & Ídem & A diario & Ídem \\
\hline Bayern Munich & 964.336 & 19 & Ídem & A diario & Ídem \\
\hline Borussia Dortmund & 828.318 & 353 & Ídem & A diario & Ídem \\
\hline Tottenham Hotspur & 785.630 & 174 & Ídem & A diario & Ídem \\
\hline Atlético de Madrid & 640.881 & 36 & Ídem & A diario & Ídem \\
\hline Inter de Milán & 494.327 & 37 & Ídem & A diario & Ídem \\
\hline AS Roma & 341.125 & 198 & Ídem & A diario & Ídem \\
\hline Schalke 04 & 165.945 & 15 & Ídem & A diario & Ídem \\
\hline Hamburgo & 111.501 & 141 & Ídem & A diario & Ídem \\
\hline Manchester United & 82.638 & 55 & Ídem & A diario & Ídem \\
\hline París Saint-Germain & 5.739 & 19 & Ídem & Cada 2 días & Ídem \\
\hline
\end{tabular}

El Real Madrid es el equipo que encabeza esta lista en cuanto al mayor número de seguidores en Twitter, seguido del F.C. Barcelona. Son los dos equipos que cuentan con más aficionados que les siguen en esta red social. Con bastante diferencia se encuentra el Galatasaray con 3.664.817 seguidores. 
Haciendo referencia al seguimiento a otras personas u organismos, es el Liverpool, con 402.756, el club que más sigue con diferencia. Tras él se encuentra el Arsenal con 163.488 y el Manchester City 57.531. Se ha podido comprobar que normalmente los equipos analizados no siguen a muchas personas u organismos en comparación con los seguidores a estos clubes.

Esta red social es la que más se actualiza, siendo numerosa la información proporcionada por el club en esta mediante un número considerable de "tuits" o entradas publicadas por el equipo de fútbol al día, siendo los clubes que más número de entradas se han registrado en el día el Tottenham Hotspur y el Inter de Milán y entre los que menos, en comparación con los demás equipos, el París Saint-Germain, que actualiza la red cada 2 días, y el Atlético de Madrid. En el caso del París Saint-Germain, este hecho de no actualizar la red tan a menudo puede repercutir en el número de seguidores, ya que es el que cierra la lista con el menor número de estos.

Todo ello nos configura una estrategia comunicativa unidireccional y pasiva por parte de los clubes y, en consecuencia, una escasez en la implicación comunicativa de los seguidores y simpatizantes.

Se ha observado que en muchos de los casos los temas tratados por los equipos en Twitter son los mismos que los tratados en Facebook. Por lo que la temática tratada en las dos redes sociales son bastante parecidas. Predominan los asuntos relacionados con la actualidad. El elemento unidireccional más utilizado es la información sobre los entrenamientos y las ruedas de prensa realizadas por los jugadores y por el cuerpo técnico. De esta manera, se priman los elementos de actualidad configurando twitter como un medio asociado al quehacer periodístico. A su vez, las acciones más activas que persiguen la implicación de los públicos tienen poca presencia en esta red social.

Tabla 4. Presencia de los clubes de fútbol en la red social Youtube.

Fuente: elaboración propia

\begin{tabular}{|c|c|c|c|c|}
\hline Youtube & $\begin{array}{c}\mathbf{N}^{\mathbf{0}} \text { de } \\
\text { Suscriptores }\end{array}$ & $\begin{array}{c}\text { Fecha de inicio en } \\
\text { la red social }\end{array}$ & $\begin{array}{c}\text { Frecuencia de } \\
\text { actualización de } \\
\text { la red }\end{array}$ & $\begin{array}{c}\text { Comentarios de } \\
\text { seguidores }\end{array}$ \\
\hline F.C. Barcelona & 1.288 .655 & $06 / 02 / 2006$ & $\begin{array}{c}\text { Apoyo al club y a los } \\
\text { jugadores. } \\
\text { Opinión, críticas y } \\
\text { sugerencias }\end{array}$ \\
\hline Real Madrid & 1.116 .541 & $05 / 04 / 2006$ & A diario & Ídem \\
\hline Chelsea & 309.430 & $28 / 02 / 2006$ & A diario & Ídem \\
\hline Juventus & 256.622 & $\begin{array}{c}\text { No se tiene } \\
\text { constancia }\end{array}$ & Cada 2 días & Ídem \\
\hline Liverpool & 226.137 & $22 / 10 / 2005$ & Cada semana & Ídem \\
\hline AC Milán & 217.855 & $\begin{array}{c}\text { No se tiene } \\
\text { constancia }\end{array}$ & Cada 3 días & Ídem \\
\hline Bayern Munich & 143.659 & $29 / 03 / 2006$ & A diario & Ídem \\
\hline Inter de Milán & 115.388 & $09 / 12 / 2005$ & A diario & Ídem \\
\hline Fenerbahçe & 94.755 & $\begin{array}{c}\text { No se tiene } \\
\text { constancia }\end{array}$ & Cada 2 ó 3 días & Ídem \\
\hline AS Roma & 73.199 & $08 / 11 / 2005$ & Cada semana & Ídem \\
\hline Tottenham Hotspur & 69.023 & $30 / 09 / 2011$ & Cada 2 ó 3 días & Ídem \\
\hline Atlético de Madrid & 44.027 & $20 / 07 / 2007$ & Cada 2 días & Ídem \\
\hline Schalke 04 & 38.996 & $\begin{array}{c}\text { No se tiene } \\
\text { constancia }\end{array}$ & Cada 3 semanas & Ídem \\
\hline
\end{tabular}




\begin{tabular}{|c|c|c|c|c|}
\hline Hamburgo & 14.945 & $\begin{array}{c}\text { No se tiene } \\
\text { constancia }\end{array}$ & Cada semana & Ídem \\
\hline Manchester United & No tiene & - & - & - \\
\hline París Saint-Germain & No tiene & - & - & - \\
\hline Manchester City & No tiene & - & - & - \\
\hline Arsenal & No tiene & - & - & - \\
\hline Borussia Dortmund & No tiene & - & - & - \\
\hline Galatasaray & No tiene & - & - & \\
\hline
\end{tabular}

El F.C. Barcelona es el equipo que cuenta con mayor número de suscriptores en Youtube, le sigue el Real Madrid y con una diferencia considerable encontramos al Chelsea. Hay 6 equipos de lista que no están presentes en esta red social.

Los primeros clubes que comenzaron a utilizar esta red social fueron en $2005 \mathrm{el} \mathrm{Li}$ verpool, AS Roma e Inter de Milán.

En Youtube se puede ver que la actualización no siempre es tan continua como en el caso de Facebook o Twitter, ya que aunque algunos suben vídeos a diario, ya encontramos otros tantos que lo hacen cada 2 ó 3 días, incluso al Liverpool que lo hace una vez en semana o al Schalke 04 cada 3 semanas, pudiendo afectar este hecho a que este equipo se encuentre el penúltimo en la lista en cuanto a número de seguidores.

En relación a los comentarios de los suscriptores, al igual que se ha comentado en otras redes sociales analizadas anteriormente, estos pueden agruparse en referencias de apoyo al club y jugadores, opiniones en general, críticas y algunas sugerencias, dependiendo de la actualidad.

Los temas tratados en los vídeos de Youtube ya no son tan variados como en Facebook o Twitter. Youtube está básicamente centrado en la transmisión de informaciones audiovisuales de actualidad como son las ruedas de prensa, informaciones sobre los entrenamientos y entrevistas ajugadores y entrenador. No existen elementos de participación ya que la tecnología de la red implica unas necesidades técnicas que dificultan la concurrencia de los públicos.

Tabla 5. Presencia de los clubes de fútbol en la red social Google+.

Fuente: elaboración propia

\begin{tabular}{|c|c|c|c|c|}
\hline Google + & $\begin{array}{c}\mathbf{N}^{\mathbf{0}} \text { de } \\
\text { suscriptores }\end{array}$ & $\begin{array}{c}\text { Fecha de inicio en la } \\
\text { red social }\end{array}$ & $\begin{array}{c}\text { Frecuencia de } \\
\text { actualización de } \\
\text { la red }\end{array}$ & $\begin{array}{c}\text { Comentarios de } \\
\text { seguidores }\end{array}$ \\
\hline F.C. Barcelona & 8.406 .263 & $\begin{array}{c}\text { No se tiene } \\
\text { constancia }\end{array}$ & $\begin{array}{c}\text { Apoyo al club y a los } \\
\text { jugadores. } \\
\text { Opinión, críticas y } \\
\text { sugerencias }\end{array}$ \\
\hline Real Madrid & 5.823 .001 & Ídem & A diario & Ídem \\
\hline Chelsea & 5.000 .030 & Ídem & Cada 2 días & Ídem \\
\hline AC Milán & 3.974 .757 & Ídem & Cada 2 días & Ídem \\
\hline Manchester City & 3.296 .611 & Ídem & A diario & Ídem \\
\hline Liverpool & 3.152 .333 & Ídem & A diario & Ídem \\
\hline Arsenal & 2.717 .522 & Ídem & A diario & Ídem \\
\hline Manchester & 2.548 .728 & Ídem & A diario & Ídem \\
\hline United & 2.163 .000 & Ídem & A diario & Ídem \\
\hline Bayern Munich & 882.415 & Ídem & A diario & Ídem \\
\hline Juventus & \multicolumn{2}{|c}{}
\end{tabular}




\begin{tabular}{|c|c|c|c|c|}
\hline $\begin{array}{c}\text { París Saint- } \\
\text { Germain }\end{array}$ & 766.522 & Ídem & A diario & Ídem \\
\hline $\begin{array}{c}\text { Atlético de } \\
\text { Madrid }\end{array}$ & 602.714 & Ídem & A diario & Ídem \\
\hline Fenerbahçe & 253.695 & Ídem & A diario & Ídem \\
\hline Schalke 04 & 161.644 & Ídem & A diario & Ídem \\
\hline Hamburgo & 133.807 & Ídem & Cada 2 días & Ídem \\
\hline $\begin{array}{c}\text { Tottenham } \\
\text { Hotspur }\end{array}$ & 99.591 & Ídem & A diario & Ídem \\
\hline Inter de Milán & 76.344 & Ídem & A diario & Ídem \\
\hline $\begin{array}{c}\text { Borussia } \\
\text { Dortmund }\end{array}$ & No tiene & - & - & - \\
\hline Galatasaray & No tiene & - & - & - \\
\hline AS Roma & No tiene & - & - & - \\
\hline
\end{tabular}

En Google+ es el F.C. Barcelona el equipo que más suscriptores cuenta, le sigue el Real Madrid y tras este el Chelsea. Cierra la lista el Inter de Milán con 76.344 seguidores de la red. En este caso son tres equipos los que no cuentan con cuentas en Google+, estos son el Borussia Dortmund, el Galatasaray y el AS Roma.

En relación a la frecuencia de actualización de esta red social por parte de los clubes de fútbol, normalmente suben contenidos a diario a excepción de algunos equipos que lo hacen cada 2 días.

La temática de las entradas en Google+ vuelve a ser casi igual de variada como las de Facebook o Twitter. Se mantiene el tono pasivo y unidireccional en la estrategia de Google+, frente a una acción activa que es muy escasa. Esto implica que los clubes de fútbol no realizan un uso adecuado de esta red social y no aprovechan las potencialidades que aporta a la estrategia de comunicación.

Tabla 6. Presencia de los clubes de fútbol en la red social Instagram

\begin{tabular}{|c|r|r|c|c|c|}
\hline Instagram & $\begin{array}{c}\text { No de } \\
\text { seguidores }\end{array}$ & $\begin{array}{c}\text { Seguimiento } \\
\text { a otros }\end{array}$ & $\begin{array}{c}\text { Fecha de } \\
\text { inicio en la } \\
\text { red social }\end{array}$ & $\begin{array}{c}\text { Frecuencia de } \\
\text { actualización de } \\
\text { la red }\end{array}$ & $\begin{array}{c}\text { Comentarios de } \\
\text { seguidores }\end{array}$ \\
\hline F.C. Barcelona & 1.715 .486 & 21 & $\begin{array}{c}\text { No se tiene } \\
\text { constancia }\end{array}$ & A diario & $\begin{array}{c}\text { Apoyo al club y a los } \\
\text { jugadores. } \\
\text { Opinión, críticas y } \\
\text { sugerencias }\end{array}$ \\
\hline Real Madrid & 1.672 .860 & 15 & Ídem & A diario & Ídem \\
\hline Manchester United & 806.225 & 22 & Ídem & A diario & Ídem \\
\hline Arsenal & 607.441 & 18 & Ídem & A diario & Ídem \\
\hline Chelsea & 540.653 & 57 & Ídem & Cada 2 días & Ídem \\
\hline Liverpool & 424.316 & 1.273 & Ídem & A diario & Ídem \\
\hline Juventus & 286.392 & 14 & Ídem & A diario & Ídem \\
\hline Galatasaray & 273.693 & 0 & Ídem & Cada 3 días & Ídem \\
\hline AC Milán & 214.500 & 16 & Ídem & Cada 3 días & Ídem \\
\hline París Saint-Germain & 147.241 & 15 & Ídem & A diario & Ídem \\
\hline Fenerbahçe & 127.355 & 15 & Ídem & A diario & Ídem \\
\hline Tottenham Hotspur & 96.257 & 22 & Ídem & A diario o cada 2 & Ídem \\
\hline Atlético de Madrid & 74.119 & 13 & Ídem & A diario & Ídem \\
\hline Schalke 04 & 40.667 & 20 & Ídem & A diario & Ídem \\
\hline Inter de Milán & 33.212 & 9 & Ídem & A diario & Ídem \\
\hline
\end{tabular}




\begin{tabular}{|c|c|c|c|c|c|}
\hline Hamburgo & 22.671 & 27 & Ídem & A diario & Ídem \\
\hline Borussia Dortmund & 3.191 & 24 & Ídem & A diario & Ídem \\
\hline Bayern Munich & No tiene & - & - & - & - \\
\hline Manchester City & No tiene & - & - & - & - \\
\hline AS Roma & No tiene & - & - & - & - \\
\hline
\end{tabular}

En Instagram es el F.C. Barcelona el club que más seguidores tiene, siguiéndole de cerca el Real Madrid y tras este se encuentra el Manchester United. Los dos primeros equipos nombrados cuentan con bastante diferencia en relación al mayor número de aficionados que les siguen en la red.

El club que de forma más significativa sigue a otras personas u organismos en esta red social es el Liverpool, al igual que en el caso de Twitter, teniendo muy de lejos el Chelsea. Los equipos que menos siguen a otros son el Galatasaray que no sigue a nadie y el Inter de Milán que solo sigue a 9.

Las páginas de estos clubes en Instagram normalmente son actualizadas a diario o cada 2 ó 3 días.

Entre los "otros temas" recogidos en las fotografías de Instagram están las fotos de los jugadores de los equipos y de antiguos jugadores que habían formado parte de estos. Siendo las fotografías más publicadas por parte de los clubes las de los partidos jugados y entrenamientos como las fotos de los aficionados en los partidos.

\section{Conclusiones}

Observamos que los clubes de fútbol con mayores ingresos del mundo desarrollan una estrategia de comunicación 2.0 en la mayor parte de las redes sociales existentes. Algunos de los clubes hicieron su adaptación casi desde el inicio de la red como es el caso de Youtube que comenzó en febrero de 2005 y en ese mismo año tres clubes ya disponían de su propio canal como son Liverpool, AS Roma e Inter de Milán. En general, muestran una adaptación adecuada a la creación de las redes sociales y a las plataformas de la comunicación digital. Sin embargo, esa adaptación formal a las redes sociales está influida por la cotidianeidad de los clubes con informaciones diarias y con una relación directa sobre los resultados deportivos en lo referente a la participación de los seguidores. Hemos encontrado una relación mimética entre resultados deportivos con incremento de participación y alabanzas, frente a una bajada de la participación y críticas intensas ante resultados negativos.

La particularidad del fútbol como espectáculo moderno le hace disponer de millones de seguidores de todo el mundo. Así, el Real Madrid posee más de diez millones de seguidores en twitter, más de un millón de suscriptores tienen el CF Barcelona en Youtube, sus ocho millones en Google + o sus casi dos millones de Instagram. Si tendemos a las diferentes redes sociales, el triunfador en entono digital sobre el número de seguidores es el FC Barcelona ya que es el club que mayor número posee en Facebook, Youtube, Instagram y Google +. Por el contrario, el Real Madrid es el club con mayor número de seguidores en twitte. Desde esta perspectiva global, dos clubes españoles (CF Barcelona y Real Madrid) triunfan en la liga de las audiencias/seguidores en redes sociales. 
La significación política, social y deportiva de los clubes les confiere un estatus de eso periodístico alto en los medios de comunicación que dedican espacio, tiempo, personal y presupuesto a la actividad diaria de los clubes. Esa presencia permanente se acrecienta por su participación en las competiciones deportivas y supone un incremento de los flujos opinativos de los seguidores.

Desde la estructura comunicativa de los clubes de fútbol las actividades generadas en las redes sociales abarcan principalmente los partidos jugados, las ruedas de prensa y las entrevistas a jugadores y al entrenador. Son las entradas más seguidas y con mayor repercusión mediática. Eso supone que la industria mediática del fútbol necesita de continuas actividades diarias para mantener la presencia mediática.

Otro elemento que caracteriza a estas empresas es una opinión excesivamente pendular que está condicionada casi exclusivamente a los resultados deportivos.

Finalmente, resaltar que los elementos de interacción con los seguidores son poco fomentados por los clubes. Entre esos elementos interactivos hemos constatado que muy pocos clubes fomentan la participación con sorteos o concursos entre los seguidores, la posibilidad de recibir fotografías de la afición o la personalización de los mensajes.

\section{Referencias bibliográficas}

ALMANSA MARTÍNEZ, Ana y CASTILLO-ESPARCIA, Antonio (2014): “Comunicación Institucional en España. Estudio del uso que los diputados españoles hacen de las TIC en sus relaciones con la ciudadanía". Revista Chasqui, Ciespal, Quito, núm. 126, pp. 22-30

ALMANSA MARTÍNEZ, Ana; FONSECA, Óscar; y CASTILLO-ESPARCIA, Antonio (2013b): "Redes sociales y jóvenes. Uso de Facebook en la juventud colombiana y española”. Comunicar, 20,40, pp. 127-135.

ALMANZA HERNÁNDEZ, Marcela (2013): “Análisis de las estrategias de comunicación transmedia de los clubes de fútbol más relevantes de Europa: FC Barcelona, Real Madrid CF, Chelsea FC y Manchester United FC." http://hdl.handle.net/10230/22197

ARCEO, Alfredo. (2003): "El fútbol y las relaciones públicas en España”. Revista Chasqui, diciembre, núm. 84, pp. 26-33

BONAUT Joseba (2007): Televisión y deporte en España (1956-1989): una perspectiva cultural. Pamplona, Universidad de Navarra.

CASTILLO-ESPARCIA, Antonio y ÁLVAREZ NOBELL, Alejandro (2014): Evaluación en Comunicación estratégica. Madrid, Mc-Graw-Hill.

CASTILLO-ESPARCIA, Antonio; ALMANSA MARTÍNEZ, Ana (2005): "Relaciones públicas y tecnología de la comunicación. Análisis de los sitios de prensa virtuales". Organicom, num. 3, Universidad de Sao Paulo, pp. 135-149.

CASTILLO-ESPARCIA, Antonio; GARCÍA PONCE, Damián; y SMOLAK LOZANO, Emilia (2013): "Movimientos sociales y estrategias de comunicación. El 
caso del 15-M y de Occupy Wall Street". Estudios sobre el Mensaje Periodístico,vol. 19, núm.1, pp. 71-89. Madrid, Servicio de Publicaciones de la Universidad Complutense.

CASTILLO-ESPARCIA, Antonio (2002): "El papel de las fuentes. Relaciones entre gabinetes de comunicación y medios de comunicación", en FERNÁNDEZ SANZ, Juan José; RUEDA LAFFOND, José Carlos; y SANZ ESTABLÉS, Carlos (Coords.).: Prensa y periodismo especializado (historia y realidad actual), actas del Congreso de "Prensa y Periodismo Especializado", celebrado del 8-10 de mayo de 2002. Guadalajara, pp. 563-572

FULCONIS, François y PACHE, Gilles (2014) "Football passion as a religion: the four dimensions of a sacred experience". Society and Business Review, vol. 9, núm. 2, pp.166 - 185

GINESTA, Xavier (2010): "Los medios propios de los clubes de fútbol españols. De la revista oficial a los canales de TDT”. Estudios Sobre el Mensaje Periodístico, vol. 16, pp. 145-166. Madrid, Servicio de Publicaciones de la Universidad Complutense.

GINESTA, Xavier \& de SAN EUGENIO, Jordi (2013): "The Use of Football as a Country Branding Strategy. Case Study: Qatar and the Catalan Sports Press". Communication \& Sport, 25 abril, doi: 10.1177/2167479513486886

GONZÁLEZ RAMALLAL, Manuel (2004): "El reflejo del deporte en los medios de comunicación en España". Revista Española de Investigaciones Sociológicas, vol. 4, núm. 4, pp. 271-279

GONZÁLEZ RAMALLAL, Manuel (2014): "Prensa deportiva e identidad nacional: España en el Mundial de fútbol de Sudáfrica 2010". Política y Sociedad, vol.54, núm. 2, pp. 337-366. http://dx.doi.org/10.5209/rev_POSO.2014.v51.n2.43077

HARO, Carmen y SAMPEDRO, Víctor (2011): “Activismo político en Red: del Movimiento por la Vivienda Diga al 15 M". Tecnokultura, vol. 8, núm. 2, pp. 167185

L'ETANG, Jacquie (2010): "Cross-cultural sport public relations and communication, in Hopwood, Mario Kitchin, Paul and Skinner, James (2010)". Sport public relations and communication, Butterwort-Heinemann, Oxford, pp. 153-175

MENESES, Guillermo Alonso y ÁVALOS GONZÁLEZ, Juan Manuel (2013): “La investigación del futbol y sus nexos con los estudios de comunicación: Aproximaciones y ejemplos". Comunicación y sociedad, vol. 20, pp. 33-64.

MINQUET, Jean Paul (2004): "Sports, football et finance". Revue Française de Gestion, num. 150, pp. 141-160.

OLABE, Fernando (2011): "Los medios de comunicación como público objetivo de los clubes de fútbol. La Liga de Fútbol Profesional en España (LFP) como caso de estudio". Razón y Palabra, agosto-octubre, vol. 16, núm. 77. 
ROLDÁN, Diego (2007): "La espontaneidad regulada. Fútbol, autoritarismo y nación en Argentina '78. Una mirada desde los márgenes". Prohistoria, año XI, número 11, Rosario, Argentina, primavera, pp. 125-147

SOTELO, Joaquín (2012): "Deporte y social media: el caso de la Primera División del fútbol español”. Historia y Comunicación Social, vol. 17, pp. 217-230

VERCIC, Dejan; VERHOEVEN, Piet; and ZERFASS, Ansgar (2014): "Key issues of public relations of Europe: Findings from the European Communication Monitor 2007-2014". Revista Internacional de Relaciones Públicas, vol. IV, num. 8, pp. 5-26, en: http://revistarelacionespublicas.uma.es/index.php/revrrpp/article /view/290/173. [Consulta: 10 de enero de 2015]

VERHOEVEN, Piet; TENCH, Ralph; ZERFASS, Ansgar; and MORENO, Ángeles (2012): "How European PR practitioners handle digital and social media". Public relations review, 38, pp. 162-164.

XIFRA, Jordi (2008): "Soccer, civil religion, and public relations: Devotional-promotional communication and Barcelona Football Club". Public Relations Review, Vol. 34, Issue 2, pp. 192-198: http://dx.doi.org/10.1016/j.pub.

Antonio Castillo Esparcia es profesor del Departamento de Comunicación Audiovisual y Publicidad de la Universidad de Málaga.

María Jesús Fernández Torres es profesora del Departamento de Comunicación Audiovisual y Publicidad de la Universidad de Málaga.

Elizabeth Castillero Ostío es investigadora de la Universidad de Málaga. 